\title{
Variational principles for thermal transport in nanosystems with heat slip flow
}

\author{
D. Jou, ${ }^{1, *}$ G. Lebon, ${ }^{2, \dagger}$ and M. Criado-Sancho ${ }^{3, *}$ \\ ${ }^{1}$ Departament de Fisica, Universitat Autonoma de Barcelona, 08193 Bellaterra, Catalonia, Spain \\ ${ }^{2}$ Department of Astrophysics, Geophysics and Oceanography, Liège University, B 4000 Liège, Belgium \\ ${ }^{3}$ Departamento de Ciencias y Técnicas Físicoquímicas, Facultad de Ciencias, UNED, Paseo Senda del Rey 9, 28040 Madrid, Spain
}

(Received 17 May 2010; published 23 September 2010)

\begin{abstract}
Some years ago, Prigogine minimum entropy production principle was generalized by Lebon and Dauby to account for the nonlocal heat transport equation derived by Guyer and Krumhansl. Here, this criterion is extended to incorporate the effects of heat slip flow along the walls. This formulation is shown to be useful for the description of steady heat flow in nanosystems, where the heat slip flow plays a decisive role.
\end{abstract}

DOI: 10.1103/PhysRevE.82.031128

PACS number(s): 05.70.Ln, 46.15.Cc, 44.05.+e

\section{INTRODUCTION}

The general importance of the boundary conditions in hydrodynamic problems, as for instance velocity slip flow on the walls, which was during many years restricted to the domain of rarefied gases $[1,2]$ has increased considerably with the fast expansion of microfluidics and nanotechnology [3-5]. This is due to the fact that at length scales close to the mean free path of the particles, the nonslip boundary condition of classical hydrodynamics, requiring that the fluid adjacent to a wall has the same velocity as the wall, is no longer valid. This effect is especially relevant in nanosystems; indeed, the boundary layer with a characteristic width of the order of the mean free path, the so-called Knudsen layer, where the slip flow is important, becomes comparable to the dimensions of the system and therefore its influence will be felt in the whole system. As a consequence, the specification of the fluid velocity at the wall requires new constitutive equations, besides the transport equations describing the flow in the bulk of the system [6-8].

The present paper is concerned with heat transport rather than with matter flow, and it pays a special attention to nonlocal heat transfer. The analysis is based on phonon hydrodynamics [9-17], in which the phonon flow is described by equations which bear a close formal analogy with the StokesBrinkman equation of hydrodynamics in resistive media, such as porous media. Phonon hydrodynamics enhances the parallelisms between fluid flow and heat flow in nanosystems; in particular, it provides a good tool to apprehend the complexities of heat transfer in small systems, which has become a central topic in current transport theory [18-22]. In a series of previous papers [23-26], we have used phonon hydrodynamics to study heat transfer in nanowires and thin layers, by taking into account heuristic phenomenological equations at the boundaries describing the phonon-wall interactions. The physics and mathematics underlying these phenomena deserve much attention and justification and has motivated the present work.

Some years ago, Lebon and Dauby [27] proposed a variational principle for the Guyer-Krumhansl equations of pho-

\footnotetext{
*david.jou@uab.es

${ }^{\dagger}$ g.lebon@ulg.ac.be

${ }^{\ddagger}$ mcriado@ccia.uned.es
}

non heat flow, but with prescribed conditions at the wall. Here, this principle is generalized in order to derive higherorder boundary conditions for the Guyer-Krumhansl's equations. This is achieved by completing the bulk entropy production functional by entropy production terms at the boundaries. Variational formulations have played a privileged role in mechanics and more generally in physics. This interest is justified, as a variational principle presents a great power of synthesis: a single equation stands indeed for a set of differential bulk equations, initial and boundary conditions. Moreover, variational methods combine the formal elegance with the practical interest to provide simpler implementations of numerical methods and to study the stability of particular solutions [27-29]. Finally, besides their power of concision and its usefulness in numerical analysis, variational formulations may be interesting from a physical conceptual point of view, especially when the functional being minimized possesses a clear physical meaning. After discussing briefly the Lebon and Dauby's variational criterion [27] and his connection with Prigogine's minimum entropy production principle [30], we focus on the boundary conditions. Our main objective is to propose an extension of Lebon and Dauby's principle by including higher-order effects and heat slip flow on the walls, which are of primary importance in the study of heat transport in nano-materials.

\section{VARIATIONAL PRINCIPLE FOR STEADY-STATE PHONON HYDRODYNAMICS}

When the mean free path becomes comparable to or longer than the size of the system, as in nanowires or thin layers or in the heat flow around nanoscopic pores, the classical Fourier equation for heat transport is no longer valid and nonlocal effects must be incorporated into the heat transfer equation. Here, we start from the Guyer-Krumhansl's equation for the heat flux [9-16,21,22],

$$
\tau \frac{d \boldsymbol{q}}{d t}+\boldsymbol{q}=-\lambda \boldsymbol{\nabla} \theta+\ell^{2}\left[\nabla^{2} \boldsymbol{q}+2 \boldsymbol{\nabla}(\boldsymbol{\nabla} \cdot \boldsymbol{q})\right],
$$

wherein $\boldsymbol{q}$ is the heat flux vector, $\theta$ the temperature, $l$ the phonon mean free path, $\lambda$ the bulk thermal conductivity, and $\tau$ the relaxation time of the heat flux, a middot means the scalar product. This expression reduces to Fourier's law by 
letting $\tau$ and $l$ to tend to zero, and accounts for nonlocal terms which are important when the spatial scale of variation of the heat flux is comparable to the mean free path. This feature is completely lacking in Fourier's equation but it becomes relevant in nanosystems, where the spatial scale of variation of the longitudinal heat flux is of the order of the radius of the nanowire. From a microscopic point of view, Guyer and Krumhansl $[9,10]$ showed that $\tau$ is the collision time $\tau_{R}$ of resistive phonon collisions (the so-called Umklapp, or momentum nonconserving collisions), and that $l^{2}$ $=(1 / 5) c_{0}^{2} \tau_{R} \tau_{N}$, with $c_{0}$ the sound velocity and $\tau_{N}$ the collision time of normal (momentum conserving) phonon collisions. When Guyer-Krumhansl's equation is coupled to the energy balance equation

$$
c \frac{d \theta}{d t}=-\nabla \cdot q
$$

with $c$ the specific heat per unit volume, one obtains a set of two equations for the two unknown quantities $\theta$ and $\boldsymbol{q}$. As the material is supposed at rest, the material time derivative $d / d t$ may be equated to the partial time derivative $\partial / \partial t$.

Establishing boundary conditions is of huge importance in physics. In classical hydrodynamics and heat conduction problems, the boundary conditions refer to the values of the velocity, shear stress, temperature, or heat flux at the boundaries, which are generally controllable quantities. The problem becomes however more acute in a layer of a thickness of the order of the mean free path-the so-called Knudsen layer- and requires a subtler description, indeed, the velocity along the walls may be different from zero-slip flowand discontinuities of temperature may arise across the wall. In contrast with classical boundary conditions, additional constitutive equations are needed to describe these effects. When the size of the system is less than the mean free path, these boundary conditions are of huge importance because the Knudsen layer pervades the whole system and contributes to the whole flow, instead of accounting only for a minute fraction of the system near the walls. In the following, we will consider slip boundary conditions over the surface of the obstacles, by analogy with results established for flows of rarefied gases [1-3]. It is usual to refer to the role of rarefaction through the so-called Knudsen number, defined as $\mathrm{Kn} \equiv l / L$, with $L$ a characteristic size of the system, as for instance the radius of a nanowire or the thickness of a thin layer.

For rarefied flows the tangential heat flow (or slip flow) $q_{w}$ on the wall of the system is given by the analogous to the Maxwell condition used for the velocity slip flow in rarefied gas dynamics or, more generally, with its second-order extension [1-3,34-36],

$$
q_{w}=C l\left(\frac{\partial q_{b}}{\partial r}\right)_{r=R}-\alpha l^{2}\left(\frac{\partial^{2} q_{b}}{\partial r^{2}}\right)_{r=R},
$$

where $C$ and $\alpha$ are dimensionless parameters accounting for the properties of the walls More explicitly, $C$ is associated to so-called specular and diffusive collisions while $\alpha$ is related to phonon backscattering against rough walls, here the coefficients $C$ and $\alpha$ are assumed to be constant. In the rarefied gas dynamics literature, the first term, applied to the velocity and not to the heat flux, is the so-called Maxwell or Navier term, and states that the tangential flux over the surface is proportional to both the mean free path and to the surface velocity gradient [31]. Although the second term is less used, it is not unknown [34-36] and was, in particular, introduced in Ref. [24] to describe the effects of the phonon collisions with the walls of nanowires.

It was shown by Lebon and Dauby [27] that the heat flux satisfying the steady Guyer-Krumhansl relation is the one that minimizes the following functional:

$$
I=\int_{V} \sigma^{s} d V+\int_{V} \gamma \nabla \cdot \boldsymbol{q} d V,
$$

$\sigma^{s}$ being the entropy production per unit time and volume and $\gamma$ a Lagrange multiplier coping with the constraint $\boldsymbol{\nabla} \cdot \boldsymbol{q}=0$ imposed by the steady energy law [Eq. (2)]. For processes described by Guyer-Krumhansl Eq. (1), the entropy production is given by [21,27],

$$
\sigma^{s}=\left(\lambda \theta^{2}\right)^{-1}\left\{\boldsymbol{q} \cdot \boldsymbol{q}+\ell^{2}\left[(\boldsymbol{\nabla} \boldsymbol{q}):(\boldsymbol{\nabla} \boldsymbol{q})^{T}+2(\boldsymbol{\nabla} \cdot \boldsymbol{q})(\boldsymbol{\nabla} \cdot \boldsymbol{q})\right]\right\},
$$

wherein a colon stands for the complete scalar product (double scalar product for tensors of order two, triple scalar product for tensors of order three, and so on), while superscript $T$ means transposition.

It can be checked that the Euler-Lagrange relations corresponding to the variational equation $\delta I(\boldsymbol{q}, \gamma)=0$ are indeed the steady expressions of Eqs. (1) and (2) when one takes the variations with respect to $\boldsymbol{q}$ and $\gamma$ and identifies $\gamma$ with twice the inverse of the absolute temperature: $\gamma=2 \theta^{-1}$; note also that the validity of the principle is subordinated to the condition that $\lambda \theta^{2}=$ constant. Since in many situations $\lambda \theta^{2}$ is not constant, below we will discuss a suitable generalization of Eq. (4) to more general cases.

The above variational principle represents a generalization of the celebrated Prigogine's minimum entropy production principle [30] whose range of application is rather limited, as it is restricted to dissipative processes (no convection) governed by linear transport equations and constant phenomenological coefficients. In the problem of heat conduction, it amounts to say that Prigogine's principle is only applicable to heat transport processes described by Fourier's law in the form $q=\Lambda \nabla \theta^{-1}$ with $\Lambda\left(=\lambda \theta^{2}\right)=$ constant, implying that the heat conductivity $\lambda$ is proportional to $\theta^{-2}$. In the cases that $\lambda$ is a constant or behaves as $\theta^{-1}$ (as assumed in Lebon and Dauby's original formulation [27]), the quantity $\sigma^{s}$ in Prigogine's formulation must be replaced by $\theta^{2} \sigma^{s}$ and $\theta \sigma^{s}$, respectively. For the sake of simplicity and comparison with earlier works, we admit in the present and next section, with Prigogine, that $\lambda \theta^{2}$ is a constant; the more general situation wherein $\lambda \theta^{2}$ is an arbitrary function of $\theta$ will be treated in Sec. IV. An essential difference between Lebon-Dauby's and Prigogine's criteria is that the expression of the entropy production is not that derived from Classical Irreversible Thermodynamics [30], namely, $\sigma^{s}=\boldsymbol{q} \cdot \boldsymbol{\nabla} \theta^{-1}$, but instead, the one obtained from extended irreversible thermodynamics [21,22]; it is worth to recall that in their original 
formulation [27] Lebon and Dauby assumed that $\lambda \theta$ is constant so that the functional submitted to variation is the total dissipated energy $\int_{V} \theta \sigma^{s} d V$ instead of the total entropy production; in addition, the heat flux was prescribed everywhere on the boundaries $A$ so that $\delta q=0$ everywhere on $A$. Here, besides taking $\lambda \theta^{2}=$ constant, the condition that $\boldsymbol{q}$ is prescribed on the surface will be relaxed and it is asked how to modify the expression of the functional [Eq. (4)] in order to find back the boundary condition (3). This will be achieved by adding a surface term related to the heat slip flow $\boldsymbol{q}_{w}$ over the surface so that the variational equation takes the form

$$
\begin{aligned}
& \delta \int_{v}\left\{\left(\lambda \theta^{2}\right)^{-1} \boldsymbol{q} \cdot \boldsymbol{q}+\left(\lambda \theta^{2}\right)^{-1} \ell^{2}\left[(\boldsymbol{\nabla} \boldsymbol{q}):(\nabla \boldsymbol{q})^{T}+2(\boldsymbol{\nabla} \cdot \boldsymbol{q})(\boldsymbol{\nabla} \cdot \boldsymbol{q})\right]\right. \\
& \quad+\gamma \boldsymbol{\nabla} \cdot \boldsymbol{q}\} d V+\delta \int_{A} l a\left(\lambda \theta^{2}\right)^{-1}\left(\boldsymbol{q}_{w} \cdot \boldsymbol{q}_{w}\right) d A=0
\end{aligned}
$$

The surface integral in Eq. (6) has been introduced to take into account that $\delta \boldsymbol{q}_{w}$ may be different from zero in a thin layer near the wall with a characteristic width $l$, the dimensionless coefficient $a$ will be identified later on. Thus, $l d A$ indicates a small volume element of thickness $l$ over the surface $d A$. From a physical point of view, this contribution is related to the rate of entropy production at the boundaries. We have added only a contribution corresponding to a tangential heat flow along the wall because we assume, for the sake of simplicity, that the wall is insulating. Removing this restriction is straightforward but cumbersome, and will not be done here as we are interested in the conceptual problems arising in the presence of slip effects, which are already nontrivial for the slip heat flow.

After taking the variations of $\gamma, \boldsymbol{q}, \boldsymbol{q}_{w}$, and integrating by parts, one recovers the steady energy law $\boldsymbol{\nabla} \cdot \boldsymbol{q}=0$ and the steady Guyer-Krumhansl expression (1) as Euler-Lagrange equations at the condition to identify $\gamma$ with twice the inverse of the temperature [1]; at the surface, one finds

$$
\int_{A} l \delta \boldsymbol{q}_{w} \cdot\left[a \boldsymbol{q}_{w}-\ln \cdot(\boldsymbol{\nabla} \boldsymbol{q})\right] d A=0
$$

which yields the Maxwell-Navier boundary condition given by the first term in Eq. (3), with $C=1 / a$. The normal unit vector $\boldsymbol{n}$ appears in the second term of Eq. (7) as a consequence of applying that the volume integral of $(\boldsymbol{\nabla} \boldsymbol{q}):(\boldsymbol{\nabla} \delta \boldsymbol{q})$ corresponding to the variation of the second term of the first integral in Eq. (6) is the volume integral of $-\delta \boldsymbol{q} \cdot \nabla^{2} \boldsymbol{q}$, leading to the nonlocal term of the Guyer-Krumhansl equation in the volume, plus the surface integral of $\delta \boldsymbol{q} \cdot(\boldsymbol{n} \cdot \nabla \boldsymbol{q})$. Along the surface, we identify $\delta \boldsymbol{q}$ with $\delta \boldsymbol{q}_{\mathrm{w}}$. This result shows that the Maxwell-Navier boundary condition is directly related to the presence of the $\boldsymbol{\nabla} \boldsymbol{q}: \boldsymbol{\nabla} \boldsymbol{q}^{T}$ term in Eq. (5). Note that the contribution arising from the variation of $\boldsymbol{\nabla} \cdot \boldsymbol{q}$ vanishes at the wall, because it leads to a term of the form $\delta(\boldsymbol{n} \cdot \boldsymbol{q})$, which is zero when the heat flux is prescribed, and in the particular case of an insulated wall, where the normal component of $\boldsymbol{q}$ on the wall is zero. If this condition is not fulfilled, the surface integral should be completed by an additional term.

\section{SECOND-ORDER FORMALISM FOR THE BOUNDARY HEAT FLOW}

In Eq. (7) we have established the first-order MaxwellNavier boundary condition for the slip heat flow. To obtain the second-order contribution introduced in Eq. (3), we must go one step ahead in the general analysis, by developing a formalism beyond Guyer-Krumhansl's equation. This is achieved by enlarging the space of the state variables with supplementary variables $Q^{(2)}, Q^{(3)}, Q^{(4)} \ldots$ representing the flux of $\boldsymbol{q}$, the flux of the flux of $\boldsymbol{q}$, etc. [21,22]. From the kinetic theory point of view, the quantities $Q^{(n)}$ represent the symmetrized higher moments of the velocity distribution, in Cartesian coordinates,

$$
\boldsymbol{q}=c^{2}(h / 2 \pi) \int \boldsymbol{k} f(\boldsymbol{k}) \boldsymbol{d} \boldsymbol{k} ; \quad \boldsymbol{Q}^{(2)}=c^{2}(h / 2 \pi) \int \boldsymbol{k} \boldsymbol{k} f(\boldsymbol{k}) \boldsymbol{d} \boldsymbol{k}
$$

with $f(\boldsymbol{k})$ designating the phonon wave-vector distribution function, $\boldsymbol{k}$ the phonon wave vector, $h$ the Planck's constant, and $c$ the sound speed in the solid; by construction, these moments are symmetric with respect to permutations of two of the indices $i, j, k, \ldots$.

The present approach is based on the hierarchy of equations for $\boldsymbol{Q}^{(1)}(=\boldsymbol{q})$ and the higher-order fluxes $\boldsymbol{Q}^{(2)}$ and $\boldsymbol{Q}^{(3)}$ which, in its simplest approach, takes the form $[21,22]$

$$
\begin{gathered}
\tau \frac{d \boldsymbol{q}}{d t}+\boldsymbol{q}=-\lambda \boldsymbol{\nabla} T+\boldsymbol{\nabla} \cdot \boldsymbol{Q}^{(2)}, \\
\tau_{2} \frac{d \boldsymbol{Q}^{(2)}}{d t}+\boldsymbol{Q}^{(2)}=-\lambda_{2}(\boldsymbol{\nabla} \boldsymbol{q})+\boldsymbol{\nabla} \cdot \boldsymbol{Q}^{(3)}, \\
\tau_{3} \frac{d \boldsymbol{Q}^{(3)}}{d t}+\boldsymbol{Q}^{(3)}=-\lambda_{3}\left(\boldsymbol{\nabla} \boldsymbol{Q}^{(2)}\right) .
\end{gathered}
$$

Expression (9a) is a generalization of Cattaneo's equation including a nonlocal contribution expressed by the term $\boldsymbol{\nabla} \cdot \boldsymbol{Q}^{(2)}$, the two next relations $(9 \mathrm{~b})$ and $(9 \mathrm{c})$ represent the simplest formulations of the relaxation equations of $Q^{(2)}$ and $Q^{(3)}$. Higher-order tensorial fluxes of order $n$, namely, $\boldsymbol{Q}^{(n)}$, may also be incorporated into the formalism and it has been shown in $[21,22]$ that this hierarchy of equations leads to a continued-fraction expansion for the thermal conductivity. In references [32,33], this continued-fraction expansion has been particularized to obtain a size-dependent thermal conductivity for nanosystems which describes the behavior of thin layers and of nanowires.

In extended irreversible thermodynamics, the entropy production corresponding to the transport Eqs. (9a) and (9c) has been checked to be given by $[21,22]$

$$
\sigma^{s}=\left(\lambda \theta^{2}\right)^{-1} \boldsymbol{q} \cdot \boldsymbol{q}+a \boldsymbol{Q}^{(2)}: \boldsymbol{Q}^{(2)}+b \boldsymbol{Q}^{(3)}: \boldsymbol{Q}^{(3)},
$$

and the corresponding entropy flux is

$$
\boldsymbol{J}^{s}=T^{-1} \boldsymbol{q}+\alpha_{1} \boldsymbol{Q}^{(2)} \cdot \boldsymbol{q}+\alpha_{2} \boldsymbol{Q}^{(3)}: \boldsymbol{Q}^{(2)},
$$

wherein $a>0, b>0, \alpha_{1}$, and $\alpha_{2}$ are coefficients dependent on temperature but whose explicit expression is not needed for the present purposes. Within the hypotheses of relaxation 
times $\tau_{1}$ an $\tau_{2}$ negligible compared to $\tau_{0}$ and weak nonlocality, which implies that $\boldsymbol{Q}^{(n)}$ is not influenced by $\boldsymbol{\nabla} \cdot \boldsymbol{Q}^{(n+1)}$, one obtains from Eqs. (9a) and (9b)

$$
Q^{(2)}=-\lambda_{2} \nabla \boldsymbol{q}, \quad Q^{(3)}=-\lambda_{3} \nabla Q^{(2)}=\lambda_{2} \lambda_{3} \nabla \nabla q .
$$

After substitution of these results in expression (10), one is led to

$$
\sigma^{s}=\left(\lambda \theta^{2}\right)^{-1}\left[\boldsymbol{q} \cdot \boldsymbol{q}+a_{1} l^{2}(\nabla \boldsymbol{q}):(\boldsymbol{\nabla} \boldsymbol{q})+a_{2} l^{4}(\boldsymbol{\nabla} \boldsymbol{\nabla} \boldsymbol{q}):(\boldsymbol{\nabla} \nabla \boldsymbol{q})\right]
$$

with the positive coefficients $a_{1}$ and $a_{2}$ given, respectively, by $a_{1}=a \lambda \theta^{2} \lambda_{2}^{2} / l^{2}, a_{2}=b \lambda \theta^{2} \lambda_{2}^{2} \lambda_{3}^{2} / l^{4}$, note that these two coefficients are not independent but related by $a_{2} / a_{1}$ $=\left(b / a l^{2}\right) \lambda_{3}^{2}$. Working within this framework, the equation for the heat flux generalizing Guyer-Krumhansl's relation can be written as

$$
\tau \frac{d \boldsymbol{q}}{d t}+\boldsymbol{q}=-\lambda \nabla T+a_{1} l^{2}\left[\nabla^{2} \boldsymbol{q}+2 \boldsymbol{\nabla}(\boldsymbol{\nabla} \cdot \boldsymbol{q})\right]+a_{2} l^{4} \nabla^{4} \boldsymbol{q}
$$

Although expression (14) bears some resemblance with Burnett's approximation, it should be emphasized that the above results have not been established from a Chapman-Enskog expansion but rather from Grad's moments expansion [2]; in that respect, the criticisms raised against instability problems in Burnetts's equations are not transposable to our results. Indeed, in the Chapman-Enskog expansion, the terms in the second spatial derivatives of the heat flux appearing in Eq. (14) are replaced by third-order spatial derivatives of temperature. This difference makes that the wave-vectordependent effective heat conductivity in Burnett's expression is of the form $\lambda(k)=\lambda\left(1-a_{1} \lambda l^{2} k^{2}\right)$, which becomes negative for high enough values of $k$, whereas in Eq. (14), the effective thermal conductivity has the form $\lambda(k)=\lambda[1$ $\left.+\left(a_{1} / \lambda\right) l^{2} k^{2}\right]$, which is always positive [21].

The variational principle generalizing Eq. (6) is now given by

$$
\begin{aligned}
& \delta \int\left\{\boldsymbol{q} \cdot \boldsymbol{q}+l^{2}\left[a_{1}(\boldsymbol{\nabla} \boldsymbol{q}):(\boldsymbol{\nabla} \boldsymbol{q})+2 a_{1}(\boldsymbol{\nabla} \cdot \boldsymbol{q})(\boldsymbol{\nabla} \cdot \boldsymbol{q})\right]\right. \\
& \left.\quad+a_{2} l^{2}(\boldsymbol{\nabla} \boldsymbol{\nabla} \boldsymbol{q}):(\boldsymbol{\nabla} \boldsymbol{\nabla} \boldsymbol{q})\right\}\left(\lambda \theta^{2}\right)^{-1} d V+\delta \int \gamma(\boldsymbol{\nabla} \cdot \boldsymbol{q}) d \boldsymbol{V} \\
& \quad+\delta \int\left(\lambda \theta^{2}\right)^{-1} \boldsymbol{q}_{w} \cdot \boldsymbol{q}_{w} l d A=0
\end{aligned}
$$

It is checked that the Euler-Lagrange equation corresponding to the volume integral is given by relation (12), whereas the surface term takes the form

$$
\int_{A} l \delta \boldsymbol{q}_{w} \cdot\left[\boldsymbol{q}_{w}-l a_{1} \boldsymbol{n} \cdot(\boldsymbol{\nabla} \boldsymbol{q})-l^{2} a_{2} \boldsymbol{n n}: \boldsymbol{\nabla} \boldsymbol{\nabla}\right] d A=0
$$

This relation is satisfied for arbitrary variations $\delta \boldsymbol{q}_{w}$ at the condition that

$$
\boldsymbol{q}_{w}=l a_{1} \boldsymbol{n} \cdot \boldsymbol{\nabla} \boldsymbol{q}+l^{2} a_{2} \boldsymbol{n} \boldsymbol{n}: \boldsymbol{\nabla} \boldsymbol{\nabla} \boldsymbol{q} .
$$

The first-order term in $\boldsymbol{\nabla} \boldsymbol{q}$ in the right-hand side is now complemented by a term of second order in $\nabla \nabla q$ which, in one-dimensional problems, reduces to $\partial^{2} q / \partial r^{2}$, this result is strictly comparable to the second term in Eq. (2). This establishes clearly the correlation between this second-order contribution and the higher-order nonlocal term in Eqs. (11) and (12).

\section{GENERALIZATION AND CONCLUDING REMARKS}

The most important results of the present work are embodied in Eqs. (6) and (13). The main drawback is that the validity of the results is subordinated to a particular, dependence of the conductivity with respect to the temperature, namely, $\lambda \sim \theta^{-2}$. This is a relevant topic because phonon heat conductivity has in general a complicated dependence on it. Here, we will present a more general formulation valid whatever the dependence of $\lambda \theta^{2}$ with $\theta$. This is achieved by substituting the functional $I$ in Eqs. (6) or (13) by the following expression:

$$
I=\int\left(\lambda \theta^{2} \sigma^{s}+\gamma \boldsymbol{\nabla} \cdot \boldsymbol{q}\right) d V+\int a l \boldsymbol{q}_{w} \cdot \boldsymbol{q}_{w} d A,
$$

with the Lagrange multiplier $\gamma$ now given by

$$
\gamma=\int_{0}^{\theta} \lambda(\xi) d \xi
$$

The price paid for this generalization is that the functional submitted to variation is no longer the entropy production but nevertheless a quantity directly related to it by the factor $\lambda \theta^{2}$. The main advantage is that the principle covers now a much wider range of realistic applications.

It is interesting to observe that the results of the present analysis bear some resemblance with these derived by Struchtrup and Torrilhon [37], on completely different grounds. Starting from Grad's thirteen-moments method, these authors calculate the entropy rate generated at the boundaries in rarefied gas flows, which is shown to be a bilinear expression in thermodynamics fluxes and forces. Using the formalism of linear irreversible thermodynamics [30], the required boundary conditions are then obtained in the form of linear flux-force relations; in particular, the boundary condition for the tangential heat flow $q_{w}$ is shown to be proportional to the second moment $\boldsymbol{Q}^{(2)}$ in agreement with our results [Eqs. (7) and (17)], after use is made of relation (12).

To summarize, we have revisited and generalized LebonDauby's variational principle [27] whose main merit was to show that the principle of minimum entropy production set originally forth by Prigogine is applicable to GuyerKrumhansl's relation. In the present paper, Lebon-Dauby's principle has been extended by the inclusion of higher-order nonlocal terms, in that respect, we refer to expressions (13) and (17). Another originality of this work is the inclusion of surface terms in the variation equation. Indeed, in LebonDauby's original formulation, it was assumed that the heat 
flux is prescribed at the boundaries. This restriction has been relaxed and we have been able to derive boundary conditions like Eq. (17) which are frequently used in microfluidics and in nanomaterials. Moreover, the fact that these boundary conditions are the necessary conditions for the entropy production being extremal provides a further physical justification of such relations which, in the past, were introduced as ad hoc conditions.

In particular, it is of interest to observe that the variational formulation of the equations provides a way to explore the connections between the boundary constitutive equations and the bulk evolution equations. Indeed, it is worth to stress that terms of first order in the mean free path $l$ in Eq. (2) are connected to second-order terms in $\nabla^{2}$ in Guyer-Krumhansl equation, terms in $l^{2}$ in the boundary conditions are associated with terms in $\nabla^{4}$ and so on. Note that Eq. (14) contains no information about the Knudsen layer; this information comes from the boundary equations for slip heat flow, as given, for instance, by Eq. (3). Indeed, in [24,26] it has been shown that Eq. (14) leads to poor predictions for the effective thermal conductivity of nanowires, which considerably improves, instead, when the slip heat flow [Eq. (3)] is taken into account.

A final remark about the philosophy underlying variational principles is in form. The Euler-Lagrange equations and the natural boundary conditions represent the necessary conditions for the functional $\boldsymbol{I}$ to be extremal; since the functional $\boldsymbol{I}$ is positive definite as a consequence of the positiveness of the entropy production, the extremum is truly a minimum. It may be argued that the proposed variational principles are ad hoc as the knowledge of the evolutions equations is needed as a preliminary to construct the expression of the entropy production, so that in some sense, the output is identified as being the input. This is not correct as the output contains more than the input, namely the natural boundary conditions which are typical of the variational equation and which are by no means given a priori. Deriving these natural boundary conditions was furthermore the main motivation for writing this paper. Moreover the methodology which is followed is not exclusive of the present work. Indeed, the Prigogine's minimum entropy production, Glansdorff-Prigogine's local potential [38], Euler's principle in hydrodynamics have been constructed from the a priori knowledge of the equations derived as Euler-Lagrange equations.

It should be stressed that a variational principle contains more information than the field equations themselves, for instance, the property that the steady state is associated to the extremum of a physical quantity. Having a variational principle presents still other advantages: concision, direct access to specific numerical methods, natural boundary conditions. As shown above, when the variational equation possesses a physical meaning, it can be applied to a wider class of processes than the original application; this was in particular verified with the minimum entropy principle whose domain of applicability was shown to extend outside the classical Fourier law.

One could have the impression that the variational principle is ad hoc, i.e., chosen to get the desired outcome because of two facts: one the one side, the need of multiplying the entropy production by the thermal conductivity and the square of absolute temperature in order to make it independent of the temperature dependence of the thermal conductivity; which goes now into the Lagrange multiplier [Eq. (19)], which has not a direct physical meaning; on the other side, the form of the surface terms. Indeed, what we have presented here is a variational property of a set of known evolution equations, rather than deriving the evolution equations from a general variational principle. Of course, the latter thing would be more general and elegant, but in any case, knowing that a given set of equations obeys some variational properties has a mathematical and practical value, and may open the way to further formulations with a clearer physical meaning.

\section{ACKNOWLEDGMENTS}

D.J. and M.C.S. gratefully acknowledge the financial support of the Dirección General de Investigación Científica of the Spanish Ministry of Science and innovation under Grant No. FIS 2009-13370-C02-01, and of the Direcció General de Recerca of the Generalitat of Catalonia, under Grant No. 2009SGR-0016. G.L. thanks the support of the WallonieBruxelles-Quebec bilateral project of collaboration 06-809 (biennial 2009-2011). We wish also to thank the anonymous referees whose remarks and suggestions have contributed to improve the presentation of the paper.
[1] D. A. Lockerby, J. M. Reese, D. R. Emerson, and R. W. Barber, Phys. Rev. E 70, 017303 (2004).

[2] N. G. Hadjiconstantinou, Microscale Thermophys. Eng. 9, 137 (2005).

[3] P. Tabeling, Introduction to Microfluidics (Oxford University Press, Oxford, 2005).

[4] H. Bruus, Theoretical Microfluidics (Oxford University Press, Oxford, 2007).

[5] S. Colin, P. Lalonde, R. Caen, Heat Transfer Eng. 25, 23 (2004).

[6] H. Struchtrup, Macroscopic Transport Equations for Rarefied
Gas Flows: Approximation Methods in Kinetic Theory (Springer, Heidelberg, 2005).

[7] F. Sharipov, Phys. Rev. E 69, 061201 (2004).

[8] V. M. Zhdanov and V. I. Roldughin, Phys. Usp. 41, 349 (1998).

[9] R. A. Guyer and J. A. Krumhansl, Phys. Rev. 148, 766 (1966).

[10] R. A. Guyer and J. A. Krumhansl, Phys. Rev. 148, 778 (1966).

[11] P. F. Meier, Z. Phys. B: Condens. Matter 8, 241 (1969).

[12] D. Jou and J. Casas-Vázquez, Physica A 163, 47 (1990).

[13] R. A. Guyer, Phys. Rev. E 50, 4596 (1994).

[14] Z. Banach and W. Larecki, J. Phys. A 38, 8781 (2005). 
[15] W. S. Jiaung and J. R. Ho, Phys. Rev. E 77, 066710 (2008).

[16] V. A. Cimmelli and K. Frischmuth, Physica B 400, 257 (2007).

[17] V. A. Cimmelli, J. Non-Equilib. Thermodyn. 34, 299 (2009).

[18] D. Y. Tzou, Macro- to Micro-scale Heat Transfer: The Lagging Behaviour (Taylor \& Francis, New York, 1997).

[19] G. Chen, Nanoscale Energy Transport and Generation (Oxford University Press, Oxford, 2005).

[20] Z. M. Zhang, Nano/microscale Heat Transfer (Mc Graw Hill, New York, 2007).

[21] D. Jou, J. Casas-Vázquez, and G. Lebon, Extended Irreversible Thermodynamics, 4th ed. (Springer, Berlin, 2010).

[22] G. Lebon, D. Jou, and J. Casas-Vázquez, Understanding Nonequilibrium Thermodynamics (Springer, Berlin, 2008).

[23] F. X. Alvarez, D. Jou, and A. Sellitto, J. Appl. Phys. 105, 014317 (2009).

[24] A. Sellitto, F. X. Alvarez, and D. Jou, J. Appl. Phys. 107, 114312 (2010).

[25] F. X. Alvarez and D. Jou, J. Appl. Phys. 103, 094321 (2008).

[26] A. Sellitto, F. X. Alvarez, and D. Jou, J. Appl. Phys. 107, 064302 (2010).
[27] G. Lebon and P. C. Dauby, Phys. Rev. A 42, 4710 (1990).

[28] G. Lebon, Variational Principles in Thermomechanics, CISME Courses Vol. 262 (Springer, Berlin, 1980), pp. 221-415.

[29] B. A. Finlayson, The Method of Weighted Residuals and Variational Principles (Academic Press, New York, 1972).

[30] I. Prigogine, Introduction to Thermodynamics of Irreversible Processes (Wiley, New York, 1961).

[31] L. Wu, Appl. Phys. Lett. 93, 253103 (2008).

[32] D. Jou, J. Casas-Vazquez, G. Lebon, and M. Grmela, Appl. Math. Lett. 18, 963 (2005).

[33] F. X. Alvarez and D. Jou, Appl. Phys. Lett. 90, 083109 (2007).

[34] C. Cercirgnani, Rarefied Gas Dynamics: From Basic Principles to Actual Calculations (Cambridge University Press, Cambridge, England, 2000).

[35] R. G. Deissler, Int. J. Heat Mass Transfer 7, 681 (1964).

[36] J. Maurer, P. Tabeling, P. Joseph, and H. Willaine, Phys. Fluids 15, 2613 (2003).

[37] H. Struchtrup and M. Torrilhon, Phys. Rev. Lett. 99, 014502 (2007).

[38] P. Glansdorff and I. Prigogine, Thermodynamics of Structures, Stability and Fluctuations (Wiley, New York, 1971). 